\title{
Preoperative Evaluation of Myometrial Invasion in Endometrial Carcinoma: Prospective Intra-individual Comparison of Magnetic Resonance Volumetry, Diffusion-weighted and Dynamic Contrast-enhanced Magnetic Resonance Imaging
}

\author{
STEFAN FELIX THIEME ${ }^{1}$, FEDERICO COLLETTINI ${ }^{1}$, JALID SEHOULI ${ }^{2}$, \\ LUCIO BIOCCA $^{1}$, ALESSANDRA LELLA $^{1}$, MORITZ WAGNER $^{1}$, \\ JUMANA ALMUHEIMID ${ }^{2}$, HELMUT PLETT ${ }^{2,3}$ and MUSTAFA ZELAL MUALLEM ${ }^{2}$ \\ ${ }^{1}$ Department of Radiology, Campus Virchow-Klinikum, Charité Universitätsmedizin Berlin, \\ Corporate Member of Freie Universität Berlin, Humboldt-Universität zu Berlin, \\ and Berlin Institute of Health, Berlin, Germany; \\ ${ }^{2}$ Department of Gynecology with Center for Oncological Surgery, \\ Charité - Universitätsmedizin Berlin, Corporate Member of Freie Universität Berlin, \\ Humboldt-Universität zu Berlin, and Berlin Institute of Health, Berlin, Germany; \\ ${ }^{3}$ Department of Gynecology and Gynecological Oncology, Kliniken Essen-Mitte, Essen, Germany
}

\begin{abstract}
Aim: The purpose of this prospective study was to compare the diagnostic performance of diffusion-weighted (DWI) and dynamic contrast-enhanced imaging (DCE) and volumetric analyses in the preoperative assessment of myometrial invasion in patients with endometrial carcinoma. Materials and Methods: Thirty-five patients with endometrial cancer underwent preoperative magnetic resonance imaging including DWI and DCE for evaluation of the depth of myometrial invasion and volumetric analyses [tumor volume $(T V)$, uterine volume $(U V)$, tumor to volume ratio $(T V R=(T V / T U) \times 100)]$. The results of the evaluations were compared to the histopathological examinations. Results: DWI and DCE showed a sensitivity and specificity in evaluating the depth of myometrial invasion of $92 \%$ and $96 \%$ and $92 \%$ and $86 \%$, respectively, while volumetric analyses showed a sensitivity and specificity of $85 \%$ and $86 \%$ (TVR cut-off $=10 \%$ ) and $69 \%$ and $100 \%$ (TVR cut-off=25\%), respectively. Conclusion: DWI and DCE are both good diagnostic tools for the preoperative assessment of myometrial invasion. From our
\end{abstract}

Correspondence to: Dr. med. Dr. (syr.) Mustafa Zelal Muallem, Charité Universitätsmedizin Berlin, Campus Virchow-Klinikum, Augustenburger Platz 1, 13353 Berlin, Germany. Tel: +49 30450664373, Fax: +49 30450564900, e-mail: mustafa-zelal. muallem@charite.de

Key Words: Myometrial invasion, endometrial carcinoma, preoperative staging, magnetic resonance imaging, MR volumetry, diffusion-weighted MRI, contrast-enhanced MRI. results and literature research, there is potential for omitting gadolinium-based contrast agents given the high diagnostic value of DWI. In our patient collective, the predictive power of volumetric analyses was lower than that of DWI.

Endometrial cancer (EC) is the most frequent gynecological malignancy worldwide with approximately 300,000 new cases every year (1). Clinical staging under-stages 13-22\% of carcinomas and, therefore, since 1988 the official International Federation of Gynecology and Obstetrics (FIGO) staging combines surgical and histological findings (2), which was again revised in 2009 (3, 4). The prognosis of EC depends upon histological type, tumor grade, stage (myometrial infiltration $>50 \%$ in apparent early stage) $(5,6)$, and lymph-node status (7-9).

Since the definition of lymph-node status allows for a precise determination of prognosis and accurate tailoring of adjuvant therapy (10), it is crucial to identify preoperative and intraoperative risk factors for recognizing the patients who may have lymph-node dissemination $(5,11-17)$. Depth of myometrial invasion is the most important risk factor in apparent early-stage EC, but it is difficult to determine preoperatively. Magnetic resonance imaging (MRI) has been shown to be the most sensitive imaging modality, with promising results $(18,19)$ and dynamic contrast-enhanced (DCE)-MRI improves the accuracy of the assessment of the depth of myometrial invasion, which can be more improved by performing of diffusion-weighted MRI (DWI) $(20,21)$.

In a recent retrospective study, Nougaret et al. evaluated the use of tumor volumetry as a tool for the initial staging of 
EC and demonstrated a positive correlation of tumor volume with deep myometrial invasion, tumor grade, and lymphovascular invasion (22).

The aim of the present study was to compare the diagnostic accuracy of endometrial tumor volumetry with that of DCE imaging and DWI for detecting deep myometrial invasion in patients with EC.

\section{Materials and Methods}

General setup. This prospective, intra-individual comparative study was carried out at a single institution. The study protocol was approved by the Institutional Ethics Board with this approval number: EA1/065/12. All patients signed an approved informed consent form before study enrollment.

Study population. Between September 2012 and April 2015, 38 patients with histopathologically confirmed EC (established by means of tissue sampling with fractional dilatation and curettage) were enrolled. Three patients were excluded from the study population due to incomplete MRI examinations. Thirty-five patients were found to be eligible for final analysis.

For the majority of patients $(n=33)$, preoperative MRI examination was performed before total hysterectomy with bilateral salpingooophorectomy. Histopathology after surgery served as the standard of reference. Curettage and histopathological analysis was performed in one 28-year-old patient who did not undergo hysterectomy in order to preserve fertility. One patient with FIGO stage II EC left the study before surgery.

MRI. All patients were examined with the same 3-Tesla system (Somatom Skyra; Siemens Healthcare, Erlangen, Germany) using an 18-channel phased-array coil. The MRI protocol included standard T1-weighted and T2-weighted turbo spin-echo, DWI and gadolinium-enhanced T1-weighted sequences. T1-weighted turbo spin-echo images were obtained in the axial plane (mean field of view: $400 \mathrm{~mm}$; echo time (TE): $21 \mathrm{~ms}$; repetition time (TR): 900 ms; number of slices: 49 ; slice thickness: $4 \mathrm{~mm}$; matrix: $448 \times 336$ ). T2-weighted turbo spin-echo images were obtained in the axial (mean field of view: $240 \mathrm{~mm}$; TE: $101 \mathrm{~ms}$; TR: $4500 \mathrm{~ms}$; number of slices: 20 ; slice thickness: $5 \mathrm{~mm}$; matrix: $448 \times 358.4$ ) and sagittal (mean field of view: $240 \mathrm{~mm}$; TE: $101 \mathrm{~ms}$; TR: $6100 \mathrm{~ms}$; number of slices: 20 ; slice thickness: $5 \mathrm{~mm}$; matrix: $448 \times 358.4$ ) planes. T2weighted images were also obtained in the axial oblique plane perpendicular to the major axis of the uterine body (mean field of view: $220 \mathrm{~mm}$; TE: $91 \mathrm{~ms}$; TR: $4960 \mathrm{~ms}$; number of slices: 30; slice thickness: $3 \mathrm{~mm}$; matrix: $220 \times 220$ ). DWI with echo-planar technique (field of view: $220 \mathrm{~mm}$; number of slices: 30; slice thickness: $3 \mathrm{~mm}$; TE: $112 \mathrm{~ms}$; TR: $8600 \mathrm{~ms}$; b-value of 0 and $800 \mathrm{~s} / \mathrm{mm}^{2}$; matrix: $220 \times 220$ ) were acquired in the axial and sagittal planes. DCE images were obtained using T1-weighted fatsuppressed volumetric-interpolated breath-hold examination (VIBE) images (field of view: $395 \mathrm{~mm}$; number of slices: 50; slice thickness: $2 \mathrm{~mm}$ TE: $2.1 \mathrm{~ms}$; TR: $4.3 \mathrm{~ms}$; matrix $256 \times 196$ ). Images were acquired in the axial, sagittal and oblique coronal planes after injection of gadobutrol (Gadovist; Bayer Health Care, Berlin, Germany) at a dose of $1 \mathrm{ml} / \mathrm{kg}$ body weight and an injection flow of $2 \mathrm{ml} / \mathrm{s}$. Images were obtained at $0,30,60,120,180$ and $300 \mathrm{~s}$ after injection of the contrast agent. In order to reduce peristalsis artifacts, intramuscular administration of butylscopolamine (20 mg) was performed prior to the MRI examination.

Image analysis. Evaluation of myometrial infiltration: MR images were anonymized and stored in a picture archiving and communication system (Centricity RA1000; GE Healthcare, Waukesha, WI, USA). One radiologist with over 8 years of experience in gynecological imaging assessed all images. The depth of myometrial invasion was scored according to the revised FIGO staging system (4) using standard anatomic images and DCE or DW images at two separate readings. The reader was blinded to the patients' names, clinical histories, and to the results of the histopathological studies. The tumor was staged as FIGO IA if the tumor infiltrated up to $50 \%$ of the myometrium and as stage FIGO IB if the tumor infiltrated more than $50 \%$ of the myometrial thickness. A cervical stromal involvement defined FIGO stage II.

Tumor volumetry: DCE and DW images were transferred to a workstation for 3D-image analysis and analyzed using a semiautomatic 3D segmentation software (Medisys; Philips Research, Suresnes, France). Tumor volume was assessed semiautomatically by outlining the lesion on each image. The total tumor volume was automatically calculated by the software. The tumor contour was defined as areas of low signal intensity compared to the homogeneously enhancing normal myometrium. The volume of the uterus was measured on the basis of axial DCE images during the equilibrium phase (180 s after injection of contrast medium).

The cervix as well as uterine fibroids were carefully excluded from measurements. The tumor:volume ratio (TVR) was calculated as follows: $\mathrm{TVR}=(\mathrm{TTV} / \mathrm{TUV}) \times 100$, where TTV was the total tumor volume and TUV the total uterine volume (22).

Surgery and histopathology. Total hysterectomy with bilateral salpingo-oophorectomy was performed in 33 out of 35 patients 1 to 7 days after MRI examination. Curettage only was performed in one young patient. One patient with clinically and imaging-evident FIGO stage II left the study without undergoing surgery. Experienced Board-certified gynecological oncologists performed all surgical procedures. Patients received once-off intravenous antibiotic prophylaxis ( $1.5 \mathrm{~g}$ cefuroxime and $0.5 \mathrm{~g}$ metronidazole) at induction and low-molecular-weight heparin $(0.3-0.4 \mathrm{ml} / 24 \mathrm{~h}$ fraxiparine) subcutaneously $12 \mathrm{~h}$ after the operation. Lymph node dissection was performed only for patients with grade 3, type IIhistopathology in the same session or in confirmed FIGO stage IB or higher after receiving the final histopathological study in a second session. Expert pathologists, who were blinded to the imaging results, performed histopathological examination of all tumors. Depth of myometrial invasion (superficial invasion, confined to the endometrium or inner half of the myometrium; deep invasion, invading the outer half of the myometrium) and the presence of cervical stromal invasion were confirmed microscopically. Patients were enrolled in a follow-up program of assessments at 3-month intervals.

Statistical analysis. Diagnostic performance of the different modalities were calculated as follows: sensitivity=true positives (TP)/all positive samples $(\mathrm{P})$; specificity=true negatives $(\mathrm{TN}) /$ all negative samples $(\mathrm{N})$; accuracy $=(\mathrm{TP}+\mathrm{TN}) /(\mathrm{P}+\mathrm{N})$; positive predictive value $(\mathrm{PPV})=\mathrm{TP} /$ all positive calls; negative predictive value $(\mathrm{NPV})=\mathrm{TN} /$ all negative calls . 


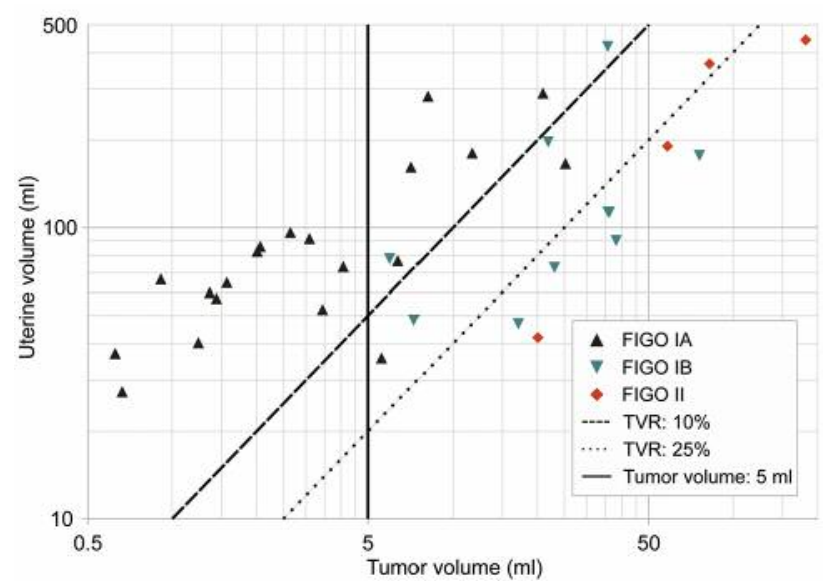

Figure 1. Distribution of different International Federation of Gynecology and Obstetrics (FIGO) stages according to tumor and uterine volume. Diagonal lines symbolize tumor:volume ratios (TVR) of $25 \%$ and $10 \%$. Tumors with a TVR higher than $25 \%$ were all FIGO stage IB or II. Most of the stage IA tumors had a TVR smaller than $10 \%$, primarily owing to their smaller tumor volumes: all tumors $<5 \mathrm{ml}$ were stage IA tumors in our collective.

\section{Results}

Baseline patient demographic and clinical characteristics were as follows: The median patient age was 60 (range=28-83) years, 28 out of 35 being at least 56 years of age $(80 \%), 11$ of them between 56 and 60 years of age. Hysterectomy and histopathological studies were performed in 33 out of 35 patients. Thirty-one patients were diagnosed with type I endometrial cancer, nine of them with squamous differentiation. In two patients, serous carcinoma was found. Fourteen out of 31 patients had a well-differentiated tumor (G1), 13 had G2 tumors (moderately differentiated) and six patients had a poorly differentiated tumor (G3). There were no undifferentiated tumors in our collective. At histopathology, 22 out of $35(62.9 \%)$ patients had a tumor with no or less than $50 \%$ myometrial invasion. One of the 22 patients had cervical stromal invasion (FIGO stage II), while the remaining 21 were staged as FIGO stage IA EC. Thirteen out of 35 patients $(37.1 \%)$ had deep myometrial invasion. Disease in three out of these 13 patients was staged FIGO stage II due to combined cervical stromal invasion; in 10 it was staged FIGO stage IB.

For detecting deep myometrial invasion, DWI achieved an accuracy, sensitivity and specificity of $94.3 \%, 92.3 \%$ and $95.5 \%$, respectively, with PPV and NPV of $92.3 \%$ and $95.5 \%$, respectively. DWI underestimated the depth of infiltration in one patient with deep myometrial infiltration and overstaged another patient with superficial myometrial infiltration at histopathology.

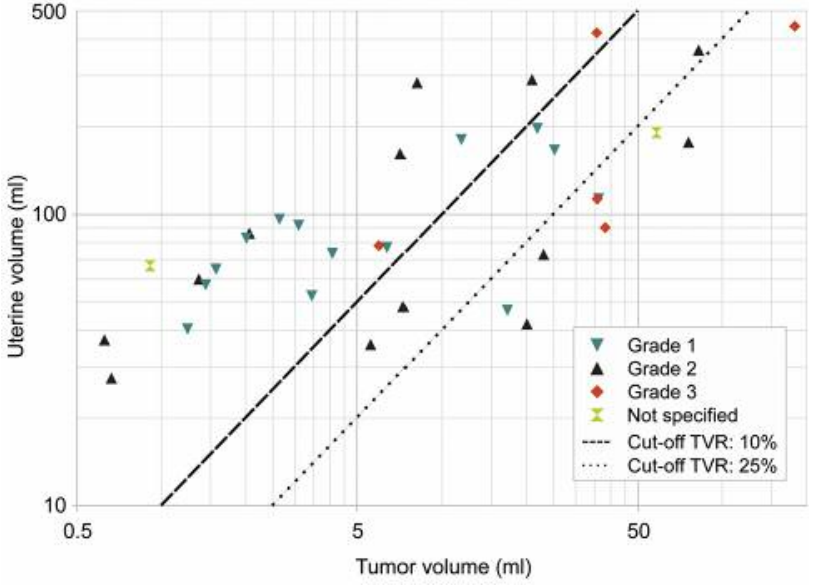

Figure 2. Distribution of histopathological grading according to tumor and uterine volume. Diagonal lines symbolize tumor:volume ratios (TVR) of $25 \%$ and $10 \%$. Unlike for regarding tumor staging, no correlation was found between tumor grade and tumor volume or tumor:volume ratio (TVR), with half of the G3 tumors having a TVR $<10 \%$ (note that one of them was not visible in imaging and therefore is not shown in the plot).

Accuracy, sensitivity and specificity of DCE-MRI were $88.6 \%, 92.3 \%$ and $86.4 \%$ with PPV and NPV of $80.0 \%$ and $95.0 \%$. DCE imaging overestimated the myometrial infiltration in three patients.

The TUV showed a large variability from 27 to $440 \mathrm{ml}$. The TTV ranged from 20 to $181 \mathrm{ml}$ for FIGO II tumors, 0 (no tumor detected in one patient) to $25 \mathrm{ml}$ for FIGO IA tumors and $6 \mathrm{ml}$ to $76 \mathrm{ml}$ for FIGO II tumors (Figure 1).

By using a TVR cut-off value of $25 \%$, deep myometrial invasion was predicted with an accuracy, sensitivity and specificity of $88.6 \%, 69.2 \%$ and $100 \%$, respectively, with a PPV and NPV of $100 \%$ and $84.6 \%$, respectively. Choosing a lower cut-off of $10 \%$ improved the weak sensitivity to $84.6 \%$ at the expense of accuracy and specificity $(85.7 \%$ and $86.4 \%)$ with PPV and NPV of $78.6 \%$ and $90.5 \%$, respectively. For a summary and comparison of test characteristics see Table I.

\section{Discussion}

Over the past decade, the role of MRI in gynecological oncology has evolved dramatically. Especially in the context of EC, MRI has been established as a valuable tool in identifying deep myometrial invasion and thereby defining the local tumor stage. Thus, MRI helps in preoperative decision-making regarding the need for lymphadenectomy in patients with EC $(23,24)$.

In recent years, DWI has been evaluated with regard to whether it can facilitate determining the depth of myometrial 
Table I. Summary of test characteristics of diffusion-weighted (DWI) and dynamic contrast-enhanced imaging (DCE) and tumor:volume ratios (TVR) evaluating deep myometrial infiltration.

\begin{tabular}{lcccc}
\hline Measure & DWI & DCE & $\begin{array}{c}\text { TVR cut-off } \\
10 \%\end{array}$ & $\begin{array}{c}\text { TVR cut-off } \\
25 \%\end{array}$ \\
\hline Accuracy (\%) & 94.3 & 88.6 & 85.7 & 88.6 \\
Sensitivity (\%) & 92.3 & 92.3 & 84.6 & 69.2 \\
Specificity (\%) & 95.5 & 86.4 & 86.4 & 100 \\
PPV (\%) & 92.3 & 80.0 & 78.6 & 100 \\
NPV (\%) & 95.5 & 95.0 & 90.5 & 84.6 \\
\hline
\end{tabular}

PPV: Positive predictive value; NPV: negative predictive value.

infiltration. DWI does not depend on the administration of gadolinium-containing contrast agents and its use is therefore independent of renal function or allergies to gadoliniumcontaining contrast agents. Therefore, several authors have proposed a prominent role of DWI in preoperative staging of EC (22, 25-26). More importantly, some studies have postulated that unlike DWI, DCE may overestimate the extent of myometrial tumor invasion due to peri-tumoral inflammation (26).

In our study, DWI compared to DCE imaging showed a slightly better diagnostic accuracy (94.3\% vs. 88.6\%), a higher specificity (95.5\% vs. 86.4\%; one $v s$. three FP samples) at the same level of sensitivity (92.3\%, one FN sample in both imaging methods in two different patients) in assessing the depth of myometrial invasion. These results match the results of the study Beddy et al. conducted comparing the performance of DWI and DCE using a 1.5 T MRI scanner. They reported accuracy, sensitivity and specificity for two readers of $90 / 85 \%$ (reader 1/reader 2), $84 / 84 \%$ and $100 / 88 \%$, respectively, in DWI compared to $71 / 79 \%, 61 / 77 \%$ and $88 / 82 \%$, respectively, in DCE and found a significant improvement in diagnostic accuracy for reader 1 using DWI (25).

Concordantly, Rechichi et al. found in a prospective study that DWI was more accurate in assessing myometrial invasion than DCE. They reported sensitivity, specificity, PPV and NPV for assessing myometrial invasion of $84.6 \%, 70.6 \%$, $52.4 \%$, and $92.3 \%$, respectively, for DWI compared to $69.2 \%$, $61.8 \%, 40.9 \%$, and $84 \%$, respectively, for DCE (26).

In the volumetric analysis, we found discrepancies from the results of Nougaret et al. who reported that a TVR of $25 \%$ or more predicted deep infiltration, G3 tumors and lymphovascular invasion (22). For TVR and tumor volume, we did not find a correlation with a higher tumor grade: half of the G3 tumors in our collective exhibited a TVR $<10 \%$, while the other half had a TVR $>25 \%$ (Figure 2). None of our patients had documented lymphovascular invasion.

Despite the finding that all tumors with a very large TVR $>25 \%$ were stage IB or II tumors, this test only had a sensitivity of $69.2 \%$, i.e. missing a large number of more advanced stage tumors. In our collective, the sensitivity of volumetric analyses was increased by lowering the cut-off to $10 \%$, reaching a sensitivity and specificity of $84.6 \%$ and $86.4 \%$, respectively, still not reaching the excellent values of DWI and DCE (Table I).

In our data set, volumetric analyses did not reliably contribute to better identification of deep myometrial infiltration given already existing information from DWI and DCE imaging. From our clinical experience, volumetric analyses are comparably time-consuming. Therefore, we conclude that in clinical practice, volumetric analyses should not be performed as routine.

Frei and co-workers calculated the pretest probability of myometrial invasion for G1, 2, and 3 tumors and concluded that MRI was reliably able to exclude deep myometrial disease in G1 and 2 disease and understaged $10 \%$ of patients with G3 disease (18). Our findings are consonant with these conclusions since one in six G3 tumors was reported FN in DWI in our study.

Taking into account previously published results, despite the small sample size of our study, we conclude that DWI and DCE-MRI are both valuable diagnostic tools for the preoperative assessment of myometrial invasion, whereas the predictive power of volumetric analyses should be interpreted with care. In future clinical routine, we see the potential for omitting gadolinium-based contrast agents given the high diagnostic value of DWI alone for our data set as well as in many previous studies $(22,25-27)$.

\section{Conflicts of Interest}

The study was supported, in part, by Bayer Health Care, Berlin, Germany.

\section{References}

1 Jemal A, Bray F, Center MM, Ferlay J, Ward E and Forman D: Global cancer statistics. CA Cancer J Clin 61(2): 69-90, 2011.

2 Shepherd JH: Revised FIGO staging for gynaecological cancer. Br J Obstet Gynaecol 96(8): 889-892, 1989.

3 Pecorelli S: Revised FIGO staging for carcinoma of the vulva, cervix, and endometrium. Int J Gynaecol Obstet 105(2): 103-104, 2009.

4 Creasman W: Revised FIGO staging for carcinoma of the endometrium. Int J Gynaecol Obstet 105(2): 109, 2009.

5 Muallem MZ, Sehouli J, Almuheimid J, Richter R, Joukhadar R and Plett H: Risk Factors of Lymph Nodes Metastases by Endometrial Cancer: A Retrospective One-center Study. Anticancer Res 36(8): 4219-4225, 2016.

6 Sorosky JI: Endometrial cancer. Obstet Gynecol 120(2): 383397, 2012.

7 Watari H, Todo Y, Takeda M, Ebina Y, Yamamoto R and Sakuragi $\mathrm{N}$ : Lymph-vascular space invasion and number of positive paraaortic node groups predict survival in node-positive patients with endometrial cancer. Gynecol Oncol 96(3): 651-657, 2005. 
8 Morrow CP, Bundy BN, Kurman RJ, Creasman WT, Heller P, Homesley HD and Graham JE: Relationship between surgicalpathological risk factors and outcome in clinical stage I and II carcinoma of the endometrium: a Gynecologic Oncology Group study. Gynecol Oncol 40(1): 55-65, 1991.

9 Chi DS, Barakat RR, Palayekar MJ, Levine DA, Sonoda Y, Alektiar K, Brown CL and Abu-Rustum NR: The incidence of pelvic lymph node metastasis by FIGO staging for patients with adequately surgically staged endometrial adenocarcinoma of endometrioid histology. Int J Gynecol Cancer 18(2): 269-273, 2008 .

10 Fanning J, Firestein S: Prospective evaluation of the morbidity of complete lymphadenectomy in endometrial cancer. Int Gynecol Cancer 8(4): 270-273, 1998.

11 Dowdy SC, Borah BJ, Bakkum-Gamez JN, Kumar S, Weaver AL, McGree ME, Haas LR, Cliby WA and Podratz KC: Factors predictive of postoperative morbidity and cost in patients with endometrial cancer. Obstet Gynecol 120(6): 1419-1427, 2012.

12 Dowdy SC, Borah BJ, Bakkum-Gamez JN, Weaver AL, McGree ME, Haas LR, Keeney GL, Mariani A and Podratz KC: Prospective assessment of survival, morbidity, and cost associated with lymphadenectomy in low-risk endometrial. cancer. Gynecol Oncol 127(1): 5-10, 2012.

13 Mariani A, Webb MJ, Keeney GL, Haddock MG, Calori G and Podratz KC: Low-risk corpus cancer: Is lymphadenectomy or radiotherapy necessary? Am J Obstet Gynecol 182(6): 1506$1519,2000$.

14 Todo Y, Watari H, Kang S and Sakuragi N: Tailoring lymphadenectomy according to the risk of lymph node metastasis in endometrial cancer. J Obstet Gynaecol Res 40(2): 317-321, 2014

15 AlHilli MM, Podratz KC, Dowdy SC, Bakkum-Gamez JN, Weaver AL, McGree ME, Kumar S, Keeney GL, Cliby WA and Mariani A: Preoperative biopsy and intraoperative tumor diameter predict lymph node dissemination in endometrial cancer. Gynecol Oncol 128(2): 294-299, 2013.

16 Kang S, Kang WD, Chung HH, Jeong DH, Seo SS, Lee JM, Lee JK, Kim JW, Kim SM, Park SY and Kim KT: Preoperative identification of a low-risk group for lymph node metastasis in endometrial cancer: a Korean Gynecologic Oncology Group study. J Clin Oncol 30(12): 1329-1334, 2012.

17 Akbayir O, Corbacioglu A, Goksedef BP, Numanoglu C, Akca A, Guraslan H, Bakir LV and Cetin A: The novel criteria for predicting pelvic lymph node metastasis in endometrioid adenocarcinoma of endometrium. Gynecol Oncol 125(2): 400403,2012
18 Frei KA, Kinkel K, Bonél HM, Lu Y, Zaloudek C and Hricak H: Prediction of deep myometrial invasion in patients with endometrial cancer: clinical utility of contrast-enhanced MR. Radiology 216(2): 444-449, 2000.

19 Faria SC, Sagebiel T, Balachandran A, Devine C, Lal C, and Bhosale PR: Imaging in endometrial carcinoma. Indian J Radiol Imaging 25(2): 137-147, 2015.

20 Freeman SJ, Aly AM, Kataoka MY, Addley HC, Reinhold C and Sala E: The revised FIGO staging system for uterine malignancies: Implications for MR imaging. Radiographics 32(6): 1805-1827, 2012

$21 \mathrm{Wu}$ WJ, Yu MS, Su HY, Lin KS, Lu KL and Hwang KS: The accuracy of magnetic resonance imaging for preoperative deep myometrium assessment in endometrial cancer. Taiwan J Obstet Gynecol 52(2): 210-214, 2013.

22 Nougaret S, Reinhold C, Alsharif SS, Addley H, Arceneau J, Molinari N, Guiu B and Sala E: Endometrial cancer: Combined MR volumetry and diffusion-weighted imaging for assessment of myometrial and lymphovascular invasion and tumor grade. Radiology 276(3): 797-808, 2015.

23 Patel S, Liyanage SH, Sahdev A, Rockall AG and Reznek RH: Imaging of endometrial and cervical cancer. Insights Imaging 1(5-6): 309-328, 2010.

24 Sahdev A and Reznek RH: Magnetic resonance imaging of endometrial and cervical cancer. Ann NY Acad Sci 1138(1): 214232, 2008.

25 Beddy P, Moyle P, Kataoka M, Yamamoto AK, Joubert I, Lomas D, Crawford R and Sala E: Evaluation of depth of myometrial invasion and overall staging in endometrial cancer: Comparison of diffusion-weighted and dynamic contrast-enhanced MR imaging. Radiology 262(2): 530-537, 2012.

26 Rechichi G, Galimberti S, Signorelli M, Perego P, Valsecchi MG and Sironi S: Myometrial invasion in endometrial cancer: Diagnostic performance of diffusion-weighted MR imaging at 1.5-T. Eur Radiol 20(3): 754-762, 2010.

27 Nougaret S, Lakhman Y, Vargas HA, Colombo PE, Fujii S, Reinhold $\mathrm{C}$ and Sala E: From staging to prognostication: Achievements and challenges of MR imaging in the assessment of endometrial cancer. Magn Reson Imaging Clin N Am 25(3): 611-633, 2017.

Received April 28, 2018

Revised June 1, 2018

Accepted June 7, 2018 